\title{
Clinical developments in the treatment of
} relapsed or relapsed and refractory multiple myeloma: impact of panobinostat, the first-in-class histone deacetylase inhibitor

\author{
This article was published in the following Dove Press journal: \\ OncoTargets and Therapy \\ 10 May 2016 \\ Number of times this article has been viewed
}

\author{
Kimberly A Redic' \\ Shannon M Hough ${ }^{2}$ \\ Erika M Price' \\ 'College of Pharmacy, University \\ of Michigan, ${ }^{2}$ Department of \\ Pharmaceutical Services, University \\ of Michigan Health System, Ann Arbor, \\ MI, USA
}

\begin{abstract}
Background: Panobinostat is a new agent for the treatment of relapsed and refractory multiple myeloma (rrMM) as part of a combination regimen. This article presents an overview of the mechanism of action, pharmacokinetics, safety, efficacy, patient care strategies, and role of the agent in treating rrMM patients.

Results: Panobinostat belongs to the class of drugs known as histone deacetylase inhibitors, and has high activity against Class I, II, and IV nonhistone deacetylases and histone deacetylases. It represents the first of its class to receive approval for use in MM, and received priority review and orphan drug status in both US and Europe, when used in combination with bortezomib and dexamethasone in the treatment of rrMM. Approval of panobinostat was based on subgroup analysis of Phase III data obtained in the PANORAMA trial program for evaluation of the combination of panobinostat, bortezomib, and dexamethasone. Additional clinical trials have continued to explore optimal dosing regimens and novel combination regimens to further clarify the optimal role of panobinostat in the arsenal of drugs for rrMM. Panobinostat has shown a manageable safety profile characterized primarily by hematologic toxicities (thrombocytopenia, neutropenia, lymphopenia, and anemia), gastrointestinal toxicities, notably diarrhea and nausea, as well as fatigue/asthenia, electrolyte abnormalities, and less commonly cardiac toxicities.

Conclusion: Panobinostat represents an important addition to the treatment armamentarium for patients with rrMM, and studies are underway evaluating its optimal dosing strategy and role in combination with other drugs used to treat this patient population.
\end{abstract}

Keywords: panobinostat, multiple myeloma, LBH589, relapsed-refractory, Farydak, HDAC

\section{Introduction}

Multiple myeloma (MM) is a plasma cell malignancy ${ }^{1}$ and is the second most prevalent form of hematologic cancer in the US. ${ }^{2}$ In 2012, there was an incidence of 62,469 cases $(0.8 \%)$, with 43,091 deaths attributed to MM worldwide. ${ }^{3} \mathrm{MM}$ is characterized by the proliferation of plasmocytomas and subsequent production of monoclonal immunoglobulin (M protein). In symptomatic MM, M proteins are present in the blood or urine and result in the classic tetrad of "CRAB" symptoms (Calcium elevation, Renal dysfunction, Anemia, and Bone destruction), manifestations of end-organ damage from deposition of $\mathrm{M}$ proteins in tissues. ${ }^{4}$ Patients are ultimately diagnosed based on an elevated serum M protein level (greater than $3 \mathrm{~g} / \mathrm{dL}$ ) and elevated plasma cells in the marrow.,6

There have been significant advancements in diagnosis and treatment of MM in the last decades, and new classes and combinations of drugs, including proteasome
Correspondence: Kimberly A Redic College of Pharmacy, University of Michigan College of Pharmacy, I500 E Medical Center Drive, Ann Arbor, MI 48I09, USA

Tel + I 7349369699

Fax + I 7346479302

Email kredic@med.umich.edu 
inhibitors and immunomodulatory drugs (IMiDs), have led to increased survival of patients. ${ }^{7}$ Current frontline therapies for newly diagnosed MM patients have improved the prognosis and extended their lifetime. Nonetheless, MM remains incurable, as nearly all patients eventually become refractory to treatment and relapse. ${ }^{6-9}$ While salvage treatments exist, relapsed or refractory MM (rrMM) patients may not respond to therapy or may be unable to tolerate therapy due to toxicities. ${ }^{8-10}$ This circumstance highlights the need for continued research for drugs with novel mechanisms of action and for new combinations of drugs with improved outcomes and safety profiles.

Panobinostat (PAN; Farydak ${ }^{\circledR}$, LBH589 Novartis Pharmaceuticals Corporation East Hanover, NJ, USA) is a member of the histone deacetylase inhibitor (HDACi) class of drugs, which has shown positive results in the MM population. PAN was approved by the US Food and Drug Administration (FDA) in February 2015 and by the European Commission in September 2015 based on Phase III subgroup analysis for use in combination therapy in the rrMM patients who had received at least two prior lines of therapy. This review focuses on the pharmacology and pharmacokinetic profile of PAN and clinical evidence to date, and highlights future research regarding the ultimate place of PAN in treatment of MM.

\section{Mechanism of action}

Histone acetyltransferases and histone deacetylases (HDACs) are the enzymes involved in the regulation of transcription within the cell. ${ }^{11}$ Histone acetylation plays a significant role in neoplasm proliferation, especially in regulation of cell cycle checkpoints, apoptosis, and cell differentiation. ${ }^{11}$ The HDACis are a class of drugs that inhibit the actions of the HDAC enzyme, ultimately allowing normal cellular control of regulatory mechanisms. In this way, HDACis promote normal immune function to decrease malignant plasma cell proliferation.

Excess histone deacetylation has downstream effects which alter gene expression through a number of mechanisms. Transcriptional machinery is unable to access DNA when chromatin is condensed secondary to the removal of acetyl groups on core histones. ${ }^{12}$ This action may decrease the expression of tumor suppressor genes. In addition, specific DNA residues may be deacetylated, altering the binding of transcription factors. This may enhance or repress DNA transcription altogether. ${ }^{12}$ Finally, HDACs are important in the regulation of chaperone proteins for other intracellular signaling pathways. ${ }^{13}$ Some key cellular proteins implicated include p53, $\alpha$-tubulin, and heat-shock protein 90. These proteins also play a role in the tumorigenesis of MM.
There are four classes of HDAC (I, II, III, and IV). The classes differ in tissue expression, localization, and protein targets. ${ }^{11}$ Several HDACis have been developed and are in varying stages of clinical trials. As a class, the HDACis affect the expression of genes and contribute to the regulation of cancer cell survival via a number of mechanisms. Most HDACis arrest the cell cycle at $\mathrm{G} 1^{14}$ and induce apoptosis by upregulation of many proapoptotic proteins and downregulation of antiapoptotic proteins such as Bcl-2. ${ }^{15}$ In addition, HDACis have a number of direct and indirect effects that contribute to oxidative damage to cellular DNA. They cause delays in mitosis by overcoming the spindle assembly checkpoint. ${ }^{16}$ HDACis also inhibit heat-shock protein 90, a cellular chaperone required for proteins involved in intracellular signaling (Raf, Her2/neu, ERK, nuclear factor kappalight-chain-enhancer of activated B cells [NF- $\mathrm{KB}]) .{ }^{17}$ They exhibit antiangiogenic effects and induce autophagy. ${ }^{16}$

Similar to other malignant cells, myeloma cells undergo epigenetic changes. ${ }^{16}$ Specific epigenetic modifications to histones can modulate the gene expression by changing the accessibility of DNA by numerous posttranslational modifications, often to the histone tails. ${ }^{18}$ These changes impact expression of the genotype, which has downstream effects on tumorigenesis. ${ }^{12}$ One such epigenetic change is regulation of acetylation of histone lysine residues by histone acetyltransferases and HDACs within myeloma cells. Hypoacetylation of histones has been observed in many cancers and is often due to overexpression of HDAC. ${ }^{19}$

Many of these mechanisms also lend to direct synergy in the treatment of malignancy when used in combination, particularly with corticosteroids or proteasome inhibitors. Combination therapy increases the activity of the HDACi in MM. ${ }^{20}$ Several possible mechanisms for this synergy exist due to the variability of HDACi targets and potency of different agents. Inhibition of proteasomes leads to the accumulation of various proteins. Proteasome inhibition in combination with aggresome inhibition by HDACis leads to significant cellular accumulation of proteins and hyperacetylation of tubulin, leading to apoptosis. ${ }^{17,20}$

Proteasome inhibition is also important in the regulation of transcription factor, NF- $\kappa \mathrm{B}$, which is constitutively active in myeloma. When NF- $\mathrm{B}$ translocates into the nucleus, it promotes cell survival with the transcription of various genes such as proinflammatory cytokines and antiapoptotic proteins such as $\mathrm{Bcl}-2 .{ }^{21}$ Inactivation of NF- $\mathrm{\kappa B}$ by deacetylase inhibition and proteasome inhibition result in synergistic apoptotic activity. Finally, as previously described, inhibition of HDAC allows for the expression of numerous tumor suppressor genes. Combination therapy with 
a proteasome inhibitor allows for a decreased breakdown of tumor suppressor proteins.

\section{Preclinical data}

PAN is a cinnamic hydroxamic acid HDACi with highly potent inhibitory activity for Class I, II, and IV nonhistone deacetylases and HDAC.22 Preclinical studies have found that PAN and other HDACis have strong antimyeloma activity. ${ }^{20}$ Much of this antitumor effect relates to modifications to intracellular activity that alter the tumor cell interactions with its microenvironment. Specifically, PAN has been noted to affect a number of apoptotic actions in cancer cells, including upregulation of the tumor suppressor gene $p 21$, leading to cell cycle arrest and apoptosis and interruption of the signaling pathway between the myeloma cells and the microenvironment which comprises the bone marrow stromal cells and extracellular matrix. ${ }^{23}$ Many key cytokines responsible for myeloma cell survival and proliferation are impacted by HDAC inhibition, including interleukin-6, vascular endothelial growth factor, and NF- $\kappa$ B. Lastly, proteasome overload occurs after a multistep pathway including inhibition of the aggresome protein pathway and the hyperacetylation of $\alpha$-tubulin. ${ }^{24}$

\section{Pharmacokinetics}

PAN is administered orally and has been formulated in capsules containing the lactate salt. PAN is slightly soluble in water, with the highest solubility in an acidic environment ( $\mathrm{pH} \mathrm{3.0).}{ }^{25}$ The absolute bioavailability is $21 \%$. Plasma concentrations (Maximum concentration [Cmax] and area under the curve 0-48 hour) were decreased when administered 30 minutes after a high-fat meal. PAN undergoes rapid absorption, with peak concentrations noted within 2 hours of oral administration, and exhibits linear pharmacokinetics when administered in doses of 10-30 mg. PAN plasma protein binding is approximately $90 \%$ and is concentration independent. It is also extensively metabolized by hepatic reduction, hydrolysis, oxidation, and glucuronidation pathways. Metabolism by cytochrome P450 (CYP)3A4 is responsible for $40 \%$ of PAN elimination. Other minor pathways include CYP2D6, CYP2C19, UGT1A1, UGT1A3, UGT1A7, UGT1A8, UGT1A9, and UGT2B4. PAN elimination is primarily fecal, with $29 \%-51 \%$ excreted in the urine. Less than $6 \%$ of the dose is eliminated as unchanged drug via either of these processes. The terminal half-life of PAN is 37 hours. However, significant interpatient variability is expected; up to twofold accumulation of PAN was seen in patients with advanced disease following chronic oral administration.

No dose adjustment is required for renal impairment. PAN is not recommended in severe hepatic dysfunction, but doses should be decreased for mild/moderate liver dysfunction. Fatal cases of gastrointestinal (GI) and pulmonary hemorrhage have been reported. Hepatotoxicity has also been reported, and liver function testing is recommended. There are currently no contraindications.

\section{Clinical trials}

A summary of the clinical trials conducted with PAN is given in Table 1.

\section{PAN as monotherapy} CLBH589B2102 (NCT00621244) was a Phase Ia/II doseescalation trial of oral PAN conducted in 176 patients with hematologic malignancies, including 12 patients with rrMM. ${ }^{26}$ Two dose-escalation regimens were evaluated (three times per week given weekly or biweekly); PAN doses ranged from 20 to $80 \mathrm{mg}$. The recommended Phase II dose for MM was $40 \mathrm{mg}$ given weekly, and the maximum tolerated dose (MTD) was PAN $60 \mathrm{mg}$ given biweekly. One rrMM patient had a partial response. Adverse events (AEs) were consistent with those seen in other trials, including GI and hematologic AEs. Overall, this trial demonstrated safety and informed dosing for the subsequent monotherapy and combination therapy.

A Phase II trial (CLBH589B2203, NCT00445068) conducted by Wolf et $\mathrm{al}^{27}$ evaluated PAN as monotherapy in 38 patients with refractory MM using PAN $20 \mathrm{mg}$ dose administered three times a week given weekly in 21-day cycles. Patients had received at least two prior lines of therapy including both an IMiD (thalidomide or lenalidomide) and bortezomib. Overall activity was deemed modest, with one partial response and one minimal response, both with good durability (19 and 28 months, respectively), but the trial was closed due to insufficient efficacy. Over $80 \%$ of patients reported GI AEs, the majority of which were grades 1-2. Hematologic AEs were the most common grade 3-4 events, and included neutropenia, thrombocytopenia, and anemia. Fatigue was also noted in $26 \%$ of patients.

\section{PAN in combination with bortezomib and dexamethasone}

While early trials failed to show sufficient activity of PAN monotherapy in the rrMM population, the toxicity profile was deemed manageable and dosing regimens were confirmed. Preclinical studies had indicated potential synergy of HDACis with bortezomib and dexamethasone with in vitro MM cell lines, and coupled with monotherapy dosing and safety data, formed the basis of the combination trials used for eventual accelerated approval of the combination regimen. ${ }^{20,24,28}$ 
Table I Summary of clinical trials of panobinostat in relapsed or relapsed/refractory multiple myeloma

\begin{tabular}{|c|c|c|c|c|c|}
\hline NCT number & Title & Phase & Drugs & Status $^{\mathrm{a}}$ & References \\
\hline NCT00532675 & $\begin{array}{l}\text { Safety study of LBH589 when given in combination } \\
\text { with lenalidomide and dexamethasone in adult } \\
\text { patients with multiple myeloma }\end{array}$ & 1 & $\begin{array}{l}\text { Panobinostat, lenalidomide, } \\
\text { dexamethasone }\end{array}$ & $\begin{array}{l}\text { Active, not } \\
\text { recruiting }\end{array}$ & 41 \\
\hline NCT0I30I807 & $\begin{array}{l}\text { Carfilzomib plus panobinostat in relapsed/ } \\
\text { refractory multiple myeloma (MM) }\end{array}$ & I & Panobinostat, carfilzomib & Recruiting & 37 \\
\hline NCT0I54943I & $\begin{array}{l}\text { A Phase I study of the combination of } \\
\text { panobinostat and carfilzomib in patients with } \\
\text { relapsed and/or refractory multiple myeloma }\end{array}$ & I & Panobinostat, carfilzomib & $\begin{array}{l}\text { Active, not } \\
\text { recruiting }\end{array}$ & 38 \\
\hline NCT01965353 & $\begin{array}{l}\text { A Phase I study of panobinostat/lenalidomide/ } \\
\text { bortezomib/dex for relapsed and relapsed/ } \\
\text { refractory multiple myeloma (PanRVD) }\end{array}$ & I & $\begin{array}{l}\text { Panobinostat, lenalidomide, } \\
\text { bortezomib, dexamethasone }\end{array}$ & Recruiting & 43 \\
\hline NCT02057640 & $\begin{array}{l}\text { MLN9708 (Ixazomib) in combination with } \\
\text { panobinostat and dexamethasone in multiple } \\
\text { myeloma }\end{array}$ & I & $\begin{array}{l}\text { Panobinostat, ixazomib, } \\
\text { dexamethasone }\end{array}$ & Suspended & 36 \\
\hline NCT00532389 & $\begin{array}{l}\text { Phase lb study of panobinostat and bortezomib } \\
\text { in relapsed or relapsed and refractory } \\
\text { multiple myeloma }\end{array}$ & $\mathrm{lb}$ & Panobinostat, bortezomib & Completed & 29 \\
\hline NCT0062I 244 & $\begin{array}{l}\text { A study of oral LBH589 in adult patients with } \\
\text { advanced hematological malignancies }\end{array}$ & $\mathrm{I} / \mathrm{II}$ & Panobinostat & Completed & 26 \\
\hline NCT009/8333 & $\begin{array}{l}\text { Panobinostat and everolimus in treating patients } \\
\text { with recurrent multiple myeloma, non-Hodgkin } \\
\text { lymphoma, or Hodgkin lymphoma }\end{array}$ & $\mathrm{I} / \mathrm{II}$ & Panobinostat, everolimus & $\begin{array}{l}\text { Active, not } \\
\text { recruiting }\end{array}$ & 47 \\
\hline NCT0I496II8 & $\begin{array}{l}\text { Study of the combination of panobinostat and } \\
\text { carfilzomib in patients with relapsed/refractory } \\
\text { multiple myeloma }\end{array}$ & $\mathrm{I} / \mathrm{II}$ & Panobinostat, carfilzomib & $\begin{array}{l}\text { Active, not } \\
\text { recruiting }\end{array}$ & 39,40 \\
\hline NCT02I457I5 & $\begin{array}{l}\text { Velcade, thalidomide, dexamethasone and } \\
\text { panobinostat treatment and panobinostat } \\
\text { maintenance in multiple myeloma (MUKsix) }\end{array}$ & $\mathrm{I} / \mathrm{II}$ & $\begin{array}{l}\text { Panobinostat, thalidomide, } \\
\text { bortezomib, dexamethasone }\end{array}$ & $\begin{array}{l}\text { Active, not } \\
\text { recruiting }\end{array}$ & 45,46 \\
\hline NCT00445068 & $\begin{array}{l}\text { A Phase II study of oral LBH589 in adult patients } \\
\text { with multiple myeloma who have received at least } \\
\text { two prior lines of therapy and whose disease is } \\
\text { refractory to the most recent line of therapy }\end{array}$ & II & Panobinostat & Terminated & 27 \\
\hline NCT0I083602 & $\begin{array}{l}\text { Efficacy of panobinostat in patients with relapsed } \\
\text { and bortezomib-refractory multiple myeloma } \\
\text { (MACSI27I) }\end{array}$ & II & $\begin{array}{l}\text { Panobinostat, bortezomib, } \\
\text { dexamethasone }\end{array}$ & Completed & 32 \\
\hline NCT0I65I039 & $\begin{array}{l}\text { Phase II, single-center, oral panobinostat } \\
\text { in combination with lenalidomide and } \\
\text { dexamethasone in multiple myeloma }\end{array}$ & II & $\begin{array}{l}\text { Panobinostat, lenalidomide, } \\
\text { dexamethasone }\end{array}$ & $\begin{array}{l}\text { Active, not } \\
\text { recruiting }\end{array}$ & 42 \\
\hline NCT02506959 & $\begin{array}{l}\text { Panobinostat combined with high-dose } \\
\text { gemcitabine/busulfan/melphalan with autologous } \\
\text { stem cell transplant for patients with } \\
\text { refractory/relapsed myeloma }\end{array}$ & II & $\begin{array}{l}\text { Panobinostat }+ \text { busulfan- } \\
\text { based conditioning regimen }\end{array}$ & Recruiting & 49 \\
\hline NCT0I023308 & $\begin{array}{l}\text { Panobinostat or placebo with bortezomib and } \\
\text { dexamethasone in patients with relapsed multiple } \\
\text { myeloma (PANORAMA I) }\end{array}$ & III & $\begin{array}{l}\text { Panobinostat, bortezomib, } \\
\text { dexamethasone }\end{array}$ & Completed & $31,33-35$ \\
\hline NCT02568943 & $\begin{array}{l}\text { An expanded treatment protocol of panobinostat } \\
\text { in combination therapy for relapsed, and relapsed } \\
\text { and refractory multiple myeloma }\end{array}$ & $\begin{array}{l}\text { Expanded } \\
\text { access }\end{array}$ & $\begin{array}{l}\text { Panobinostat, bortezomib, } \\
\text { dexamethasone }\end{array}$ & Recruiting & 51 \\
\hline
\end{tabular}

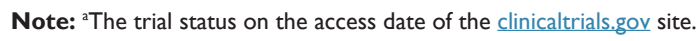

\section{Early phase trials}

A Phase Ib trial (CLBH589B2207, NCT00532389) was conducted to determine the MTD of PAN in combination with bortezomib and dexamethasone (PAN-BTZ-DEX) ${ }^{29}$ In this trial, rrMM patients $(n=62)$ were treated in dose escalation and expansion phases. In the dose-escalation phase, patients $(\mathrm{n}=47)$ received PAN starting at $10 \mathrm{mg}$ three times a week given weekly, in combination with bortezomib starting at $1 \mathrm{mg} / \mathrm{m}^{2}$ two times a week (days $1,4,8$, and 11 of a 21 -day cycle), with or without dexamethasone $20 \mathrm{mg}$ on the days of and after bortezomib doses. In the dose-expansion portion, patients $(\mathrm{n}=15)$ received a regimen of PAN $20 \mathrm{mg}$ three times 
per week for weeks 1 and 2, bortezomib $1.3 \mathrm{mg} / \mathrm{m}^{2}$ two times per week (days 1, 4, 8, and 11), and dexamethasone $20 \mathrm{mg}$ on the days of and after bortezomib doses given in 21-day cycles.

Overall response rate (ORR) in the escalation phase was $45 \%$ in all patients and $53 \%$ in those who received the final MTD of the combination. At least a very good partial response was achieved in $20 \%$ of patients. Median duration of the response was 138 days. The ORR in the expansion phase was $73 \%$ (26\% for bortezomib-refractory patients), and the median duration of response was 159 days. Similar to previous monotherapy studies, grade 3 or 4 AEs included thrombocytopenia ( $85.1 \%)$, neutropenia $(63.8 \%)$, and asthenia $(29.8 \%)$ in the escalation phase, and thrombocytopenia (66.7\%), neutropenia (46.7\%), and fatigue $(20.0 \%)$ in the expansion phase.

\section{Phase II: PANORAMA 2}

Based on the promising safety data from monotherapy and Phase Ib combination therapy trials, the PANORAMA program was developed to further evaluate the safety and efficacy of the PAN-BTZ-DEX combination regimen. ${ }^{30}$ PANORAMA 2 was a single-arm, two-stage Phase II study in rrMM patients based on the MTD dose from the $1 \mathrm{~b}$ study. PANORAMA 1 was a randomized, placebo-controlled study of the combination, evaluating the progression-free survival (PFS). ${ }^{31}$

PANORAMA 2 (CLBH589DUS71, NCT01083602) included 55 heavily pretreated patients who had received at least two (median $=4$ ) prior lines of therapy, including at least one IMiD, and who were refractory to bortezomib. ${ }^{32}$ Most patients $(73 \%)$ had progressed while on their last bortezomib regimen. In the first phase of treatment, patients were treated for up to eight 3-week cycles of PAN $20 \mathrm{mg}$ three times per week for weeks 1 and 2, bortezomib $1.3 \mathrm{mg} / \mathrm{m}^{2}$ two times per week on weeks 1 and 2, and dexamethasone $20 \mathrm{mg}$ four times a week on the days of and after bortezomib doses. The third week of each cycle was treatment free. Patients who showed clinical benefit $(n=17)$ advanced to the second treatment period, in which they received 6-week cycles of PAN $20 \mathrm{mg}$ three times per week for weeks 1, 2, 4, and 5; bortezomib $1.3 \mathrm{mg} / \mathrm{m}^{2}$ once per week on weeks 1,2 , 4, and 5; and dexamethasone $20 \mathrm{mg}$ four times per week on the days of and after bortezomib doses.

ORR with at least partial response was achieved in $34.5 \%$ of patients. Clinical benefit, defined as at least minimal response, was seen in $52.7 \%$ of patients. No patients achieved complete response (CR), but one patient achieved near CR (nCR). AEs of any grade and regardless of attribution included diarrhea $(70.9 \%)$, fatigue $(69.1 \%)$, thrombocytopenia $(65.5 \%)$, nausea $(60 \%)$, and anemia (47.3\%). The most common grade 3-4 AEs included thrombocytopenia $(63.6 \%)$, diarrhea $(20 \%)$, fatigue $(20 \%)$, anemia $(14.5 \%)$, and neutropenia (14.5\%). Platelet levels recovered quickly during the treatment-free weeks in each phase, and no patients discontinued treatment due to thrombocytopenia. Responses were durable, with median duration of response of 6 months, and PFS of 5.4 months with median overall survival (OS) of 17.5 months. Notably, patients who had not received bortezomib in their most recent line of treatment had an ORR of $42.9 \%$.

\section{Phase III: PANORAMA I}

The PANORAMA 1 trial (CLBH589D2308, NCT01023308) was conducted in 768 rrMM patients. ${ }^{31}$ This Phase III randomized, multicenter, placebo-controlled study was conducted based on the promising results obtained in the PANORAMA 2 trial, and consisted of two treatment phases. Nearly half of the patients ( $49 \%$ in the PAN-BTZ-DEX arm) had received two or more prior lines of therapy, including a large number of lines which contained bortezomib or IMiDs as single or combination regimens. In the first phase, patients were randomly assigned (1:1) and stratified based on number of prior lines of therapy and the previous use of bortezomib, to receive either PAN-BTZ-DEX or placebo-BTZ-DEX in up to eight 3-week cycles. Of those patients enrolled in the PAN-BTZ-DEX arm $(n=387), 169$ completed the first treatment period and 102 subsequently completed treatment period 2. PAN was administered three times a week, and bortezomib on days 1, 4, 8, and 11. Dexamethasone was given on the days of and after bortezomib doses. Those patients who demonstrated clinical benefit continued to a second treatment phase comprising four 6-week cycles, with a similar regimen, except that bortezomib was dosed on days $1,8,22$, and 29. The primary outcomes were overall safety and efficacy defined as PFS and $\mathrm{nCR} / \mathrm{CR}$ rates, and the results were analyzed for the two different treatment phase $^{31}$ as well as by the total duration of treatment. ${ }^{33}$

Median PFS was significantly longer in the PAN-BTZDEX arm compared to the placebo arm (12.0 vs 8.1 months, $P<0.0001)$. Median OS of patients was 40.3 months in the PAN-BTZ-DEX arm compared to 35.8 months in the placebo group; this result did not reach statistical significance. ${ }^{34}$ Of note, a higher percentage of patients in the placebo arm received post-study therapy, which may have confounded these OS results. However, the percent of patients with $\mathrm{nCR}$ 
or CR was significantly higher in the PAN group compared to placebo $(27.6 \%$ vs $15.7 \%, P=0.00006) .{ }^{33}$

An analysis of patient outcomes by prior treatments found that PFS benefit was greater in patients receiving two or more prior therapies that included bortezomib and an IMiD, and this subgroup analysis formed the basis for the accelerated approval of PAN. In this subgroup analysis, the median OS of the patients in the PAN-BTZ-DEX arm was 25.5 months compared to 19.5 months in the placebo group. ${ }^{34}$

Subsequent analyses also indicate that those patients who were able to tolerate and receive a longer duration of treatment in the PAN-BTZ-DEX arm also had a longer PFS. Those patients who completed the first treatment period had an overall PFS of 14.65 months, compared to a PFS of 17.64 months for those patients who subsequently completed the second treatment phase. ${ }^{35}$

From a safety perspective, $36 \%$ of patients in the PANBTZ-DEX arm discontinued treatment due to AEs, compared to $20 \%$ in the placebo arm. ${ }^{31}$ Grade $3 / 4$ AEs occurred in $96 \%$ of patients who received PAN-BTZ-DEX regimen versus $82 \%$ in the placebo group; the overall rate of grade $3 / 4$ AEs $(>20 \%)$ in the PAN-BTZ-DEX arm, regardless of attribution, included thrombocytopenia (68\%), diarrhea (25.5\%), fatigue/asthenia (23.9\%), lymphopenia (54\%), and neutropenia $(35 \%)$. For patients completing both treatment periods, there was a higher rate of grade 3/4 AEs in the first treatment period versus the second treatment period (thrombocytopenia: $47.1 \%$ vs $5.9 \%$; diarrhea: $25.5 \%$ vs 8.8\%; fatigue: $19.6 \%$ vs $5.9 \%$ ). Serious AEs were reported in $60 \%$ of the PAN-BTZ-DEX group compared to $42 \%$ in the placebo group, and included pneumonia (18\%), diarrhea (11\%), thrombocytopenia (7\%), fatigue $(6 \%)$, and sepsis $(6 \%)$. Analysis of platelet recovery during treatment showed that for both arms, median platelet counts returned to baseline by the start of each subsequent cycle. ${ }^{31}$

Overall, duration of treatment was shorter and frequency of AEs was greater in the PAN-BTZ-DEX group compared to the placebo group. However, for those subjects who completed both treatment periods, the incidence of new or worsening AEs was decreased in the second treatment period compared to the first. Secondary analysis of the data suggests that managing AEs in the first treatment period, so that patients can complete both treatment periods, may increase the duration of response and overall benefit. ${ }^{31}$

The PANORAMA program was notable for showing a benefit for the three-drug combination regimen in the rrMM population who had received prior therapy, and for further characterizing the toxicity profile of the combination.
The AEs noted in these trials were consistent with those in early phase trials, and were manageable with appropriate supportive care measures or dose reductions. ${ }^{30}$ In the landscape of available treatment options and outcomes in the rrMM population, the PANORAMA results, particularly the subgroup analysis in PANORAMA 1, formed the basis for the priority review and approvals of this combination by the US FDA and the European Commission.

\section{PAN in other combination regimens}

Based on the novel mechanism of action of the HDACis and the promising results of the PANORAMA program, it has been speculated that PAN in combination with other drugs and drug classes may also show benefit in the treatment of MM.

\section{Combination with other proteasome inhibitors: ixazomib}

A Phase I trial of PAN in combination with the oral proteasome inhibitor, ixazomib (MLN9708), and dexamethasone was conducted in eleven heavily pretreated patients to determine the tolerability of the combination (NCT02057640). ${ }^{36}$ Two dose levels of ixazomib ( 3 and $4 \mathrm{mg}$ ) were given on days 1,8 , and 15 with PAN $20 \mathrm{mg}$ on days 1, 3, 5, 15, 17, and 19 and dexamethasone $20 \mathrm{mg}$ on days $1,2,8,9,15$, and 16 . The combination was well tolerated; no dose reductions were required and no serious AEs were observed. A Phase II trial using the ixazomib $4 \mathrm{mg}$ dose in the combination regimen is anticipated.

\section{Combination with other proteasome inhibitors: carfilzomib}

A Phase I/Ib study of the combination of PAN and carfilzomib in rrMM patients is underway to determine the MTD and tolerability of the two-drug combination regimen (NCT01301807). ${ }^{37}$ The PAN starting dose was $15 \mathrm{mg}$ given on 3 days of weeks 1-2 plus carfilzomib starting at $20 \mathrm{mg} / \mathrm{m}^{2}$ intravenously on days $1,2,8,9,15$, and 16 of a 28 -day cycle. Results are pending.

An additional Phase I study of the combination was conducted to identify the MTD of an alternate dosing regimen of PAN given three times weekly for 3 weeks in combination with carfilzomib (NCT01549431). ${ }^{38}$ The MTD was defined as PAN $20 \mathrm{mg}$ three times a week for 3 weeks and carfilzomib $\left(20 \mathrm{mg} / \mathrm{m}^{2}\right.$ for first cycle, then escalated to $36 \mathrm{mg} / \mathrm{m}^{2}$ in subsequent cycles) given on days $1,2,8,9,15$, and 16 of a 28 -day cycle. All patients $(\mathrm{N}=20)$ had $\mathrm{rrMM}$ and had received a median of four prior lines of therapy. The most common grade $3 / 4$ toxicities included 
anemia (35\%), thrombocytopenia (35\%), neutropenia (30\%), and fatigue/asthenia (15\%). One patient had grade 3 diarrhea uncontrolled by medical management.

Another Phase I/II dose-escalation and -expansion trial was conducted evaluating another combination regimen of PAN and carfilzomib (NCT01496118). ${ }^{39}$ In this trial, PAN was given to patients with $\mathrm{rrMM}$ who had received one to nine prior lines of therapy $(\mathrm{N}=80)$. PAN was administered three times weekly every other week combined with carfilzomib. In the dose-escalation portion of the trial, MTD was not established, but the planned expansion dose was established as Dose Level 4 (DL4: PAN $30 \mathrm{mg}$ on days 1, 3, 5, 15,17 , and 19 and carfilzomib $20 / 45 \mathrm{mg} / \mathrm{m}^{2}$ given on days 1 , $2,8,9,15$, and 16 of a 28 -day cycle).

The expansion design was modified to include Dose Level 6 (DL6) using PAN $20 \mathrm{mg}$ and carfilzomib 20/56 mg/m² doses given on the same schedule as the alternate expansion dose..$^{40}$ Preliminary results indicate an ORR of $75 \%$ for all patients and $72 \%$ for DL4 patients versus $84 \%$ for DL6 patients. Median PFS was $13.5 \%$ (DL4) and $18.7 \%$ (DL6) to date; median OS has not been achieved. There were no significant differences in AEs between the two dosing levels. The most common AEs across all grades were thrombocytopenia (73\%), nausea $(69 \%)$, diarrhea (64\%), and fatigue/asthenia (51\%).

\section{Combination with IMiDs: lenalidomide}

A Phase I dose-escalation study of PAN combined with lenalidomide and dexamethasone was conducted in rrMM patients with at least one prior line of therapy $(\mathrm{N}=46$, NCT00532675). ${ }^{41}$ No new AEs were identified, and the results informed the dosing used in a subsequent Phase II trial in rrMM patients $(\mathrm{N}=27, \mathrm{NCT} 01651039) .{ }^{42}$ In the Phase II trial, PAN 20 mg was given on days 1, 2, 5, 15, 17, and 19, lenalidomide $25 \mathrm{mg}$ on days 1-21, and dexamethasone $40 \mathrm{mg}$ was given on days 1,8 , and 15 . This all-oral regimen appeared to be well tolerated, with expected hematologic AEs noted, but no serious GI AEs reported.

A Phase I study of PAN given with lenalidomide, bortezomib, and dexamethasone (PanRVD) in rrMM patients is in progress to establish the MTD of this combination (NCT01965353). ${ }^{43}$ PAN is given on days $1,3,5,8,10$, and 12 ; lenalidomide is given on days $1-14$; bortezomib is administered subcutaneously on days 1, 4, 8, and 11; and dexamethasone is given on the days of and following bortezomib. Target enrollment is $\mathrm{N}=28 \mathrm{rrMM}$ patients who have received two or more prior lines of therapy. In addition, the PanRVD regimen is also under investigation in a dose-escalation study in the newly diagnosed MM population (NCT01440582). ${ }^{44}$
This frontline trial will provide further clarity on the MTD and tolerability of the combination.

\section{Combination with IMiDs: thalidomide}

A Phase I/IIa dose-escalation and -expansion study of PAN given with thalidomide, bortezomib, and dexamethasone (PanVTD) is in progress to establish the efficacy and safety of this combination (MUK-Six, NCT02145715). In the Phase I portion, patients with rrMM who had received one to four prior lines of therapy were treated with PanVTD with escalating doses of PAN. ${ }^{45}$ The recommended dose for the regimen was determined to be PAN $20 \mathrm{mg}$ on days $1,3,5,8$, 10 , and 12 ; bortezomib $1.3 \mathrm{mg} / \mathrm{m}^{2}$ subcutaneously on days 1 and 8; thalidomide $100 \mathrm{mg}$ daily; and dexamethasone $20 \mathrm{mg}$ on the days of and following bortezomib. ${ }^{45}$ In the Phase IIa portion, patients $(\mathrm{N}=46)$ were treated at the recommended dose every 3 weeks for up to 16 cycles followed by a year of PAN maintenance. ${ }^{46}$ Transplant-eligible patients received a minimum of six cycles. Preliminary results show an ORR ( $\geq$ partial response $[\mathrm{PR}]$ ) for patients who received at least one dose of PAN of $91.3 \%$. Treatment was generally well tolerated. Grade 3/4 AEs reported across both phases included neutropenia (24.6\%), hypophosphatemia (19.3\%), thrombocytopenia (14.1\%), and diarrhea (10.5\%). These AEs were at a lower incidence than those reported in PANORAMA 2; of note, bortezomib dosing was less frequent and given subcutaneously in the MUK-Six trial. These preliminary results are promising from both an efficacy and tolerability perspective and further support a potential role of PAN in long-term maintenance therapy.

\section{Combination with mammalian target of rapamycin inhibitors}

An early-stage trial evaluating the combination of PAN with the mammalian target of rapamycin inhibitor everolimus currently has results pending (NCT00918333). ${ }^{47}$ In this Phase $\mathrm{I} / \mathrm{Ib}$ dose-escalation study in relapsed or refractory lymphomas or rrMM, doses of both drugs were escalated as tolerated to MTD. PAN was given three times weekly every other week in combination with everolimus given daily.

\section{Combination therapy with monoclonal antibodies (MAbs)}

Preclinical work has demonstrated that HDACis may enhance the efficacy of anti-CD20 MAbs by upregulating CD20 levels. An in vitro study with HDAC pan-inhibitors (trichostatin A and vorinostat) demonstrated promising results for combining this class with anti-CD38 MAbs, such as those 
targeted by daratumumab (approved by the US FDA in November 2015). ${ }^{48}$

\section{Conditioning regimens for autologous stem cell transplant (ASCT)}

A Phase II trial examining the role of PAN combined with high-dose gemcitabine/busulfan/melphalan with ASCT in rrMM is currently enrolling patients (NCT02506959). ${ }^{49} \mathrm{PAN}$ is administered as part of the conditioning regimen $(20 \mathrm{mg}$ on days -9 to -2 ). Primary outcomes include PFS and overall safety profile of the combination conditioning regimen. The results of this and additional future studies will be important for clarifying the optimal role of PAN in relation to ASCT.

\section{Overall safety and patient management strategies}

Taken as a whole, the clinical evidence to date suggests that PAN has a generally acceptable tolerability profile. Recommended dosing is $20 \mathrm{mg}$, taken orally once every other day as three doses per week (on days 1, 3, 5, 8, 10, and 12) of weeks 1 and 2 of each 21-day cycle. The most common adverse reactions (incidence of at least 20\%) in clinical studies are diarrhea, fatigue, nausea, peripheral edema, decreased appetite, pyrexia, and vomiting. The most common nonhematologic laboratory abnormalities (incidence $\geq 40 \%$ ) are hypophosphatemia, hypokalemia, hyponatremia, and increased creatinine. The most common hematologic laboratory abnormalities (incidence $\geq 60 \%$ ) are thrombocytopenia, lymphopenia, leukopenia, neutropenia, and anemia. ${ }^{24}$ Due to overlapping toxicities of drugs studied in combination with PAN, judicious use of dose reductions and interruptions coupled with sound medical management are important for increasing duration of therapy, improving clinical outcomes, and providing better quality of life for the patient.

US prescribing information includes boxed warnings for diarrhea. ${ }^{24}$ Further subanalysis of the results of the PANORAMA trials has shown that diarrhea occurred primarily in the first four cycles of the combination regimen, that incidence plateaued around the sixth cycle, and that most patients experienced complete resolution by the end of treatment. ${ }^{50}$ These results suggest that prompt intervention with supportive medications and/or judicious use of dose holds and adjustments can be used to allow patients to remain on treatment. The expanded access trial that was developed to provide access to PAN prior to US FDA approval PANobinostat EXpanded access trial (PANEX, NCT02568943) utilized the PAN-BTZ-DEX regimen from PANORAMA $1 .{ }^{51}$ In the subgroup of patients who received bortezomib subcutaneously, the rate of grade 3/4 diarrhea was $11.8 \%$ compared to $25 \%$ reported in PANORAMA 1 , suggesting that the route of bortezomib administration may impact the tolerability of the combination regimen. Additional studies may further clarify approaches for minimizing the incidence of diarrhea.

Because a small number of patients who received PAN had severe and fatal cardiac events, including ischemic events, electrocardiogram changes, and severe arrhythmias, US prescribing information also includes a boxed warning regarding increased risk of cardiac toxicity. ${ }^{24}$ In PANORAMA 1, incidence of cardiac events for the PAN-BTZ-DEX arm compared to the placebo arm was as follows: cardiac ischemia $4 \%$ versus $1 \%$, arrhythmias $12 \%$ versus $5 \%$, ST-segment depression in $22 \%$ versus $4 \%$, and T-wave abnormalities $40 \%$ versus $18 \% .{ }^{4}$ PAN should not be initiated in patients with recent history of cardiac events, and concomitant use of medications known to prolong QT interval is not recommended. A baseline electrocardiogram is recommended prior to starting therapy for all patients. A summary of AEs is listed in Table 2.

Potential drug-drug interactions with PAN are abundant due to its metabolism through the various CYP pathways. US

Table 2 Adverse Events of Panobinostat ${ }^{31}$

\begin{tabular}{ll}
\hline & $\begin{array}{l}\text { Incidence* } \\
\text { All Grades (Grade 3/4) }\end{array}$ \\
\hline Hematologic & \\
Thrombocytopenia & $98 \%(68 \%)$ \\
Lymphopenia & $83 \%(54 \%)$ \\
Leukopenia & $81 \%(24 \%)$ \\
Neutropenia & $75 \%(35 \%)$ \\
Anemia & $62 \%(18)$ \\
Non-Hematologic Laboratory & \\
Hyperbilirubinemia & $21 \%(1 \%)$ \\
Hypophosphatemia & $64 \%(20 \%)$ \\
Hyponatremia & $49 \%(13 \%)$ \\
$\quad$ Elevated serum creatinine & $41 \%(1 \%)$ \\
Diarrhea & $68 \%(25 \%)$ \\
Fatigue & $57 \%(24 \%)$ \\
Nausea & $36 \%(5 \%)$ \\
Vomiting & $26 \%(7 \%)$ \\
Decreased appetite & $28 \%(3 \%)$ \\
Peripheral edema & $29 \%(2 \%)$ \\
Pyrexia & $26 \%(1 \%)$ \\
ST-segment depression & $22 \%$ \\
T-wave abnormalities & $40 \%$ \\
\hline Not
\end{tabular}

Note: *Side effects reported in combination with bortezomib and dexamethasone. Reprinted from Lancet Oncol, I5/II, San-Miguel JF, Hungria VT, Yoon SS, et al, Panobinostat plus bortezomib and dexamethasone versus placebo plus bortezomib and dexamethasone in patients with relapsed or relapsed and refractory multiple myeloma: a multicenter, randomized, double-blind phase 3 trial, II95-1206, Copyright (2014), with permission from Elsevier. 
prescribing information includes recommendations to reduce the dose when used in combination with strong CYP3A4 inhibitors and avoid PAN in patients taking strong CYP3A4 inducers or sensitive CYP2D6 substrates. ${ }^{24}$

\section{Future directions}

Despite advances in treatment of rrMM and positive findings related to PAN in the pretreated population, it is challenging to predict an individual patient response to treatment. The relative lack of head-to-head comparisons of treatment options has led to the use of novel statistical techniques to predict the relative effectiveness of various regimens.

An indirect treatment comparison of multiarm, randomized controlled trials conducted in rrMM patients (including PANORAMA 1) was conducted by Richardson et $\mathrm{al}^{52}$ using the Markov Chain Monte Carlo simulation method. In addition, results from a network meta-analysis of rrMM trials reporting PFS, OS, or time to progression determined odds ratio for pairwise comparisons of regimens, including PAN given with bortezomib and dexamethasone..$^{53}$ Both frameworks offer novel approaches that may be coupled with patient-specific factors, in order to better predict individual patient response to specific PAN combinations.

Because MM is characterized by genetic clonal heterogeneity, strategies to predict patient response using genomic profiling are important topics for future research. Recent in vitro studies underscore the importance of elucidating the mutation profile and sequence of molecular events in the treatment of MM. ${ }^{54}$ The umbrella trial design is intended to facilitate enrollment and analysis of results for malignancies with heterogeneity in genomic mutations. Based on the variety of mutations seen in MM, large umbrella trials with genomic profile matching to single-drug or combination regimen therapies are anticipated that include both frontline and salvage therapies. Based on PAN's demonstrated role in vitro against MM cell lines with overexpression of specific HDAC isoenzymes, ${ }^{20,27}$ it will be important to include PAN in trials designed to optimize therapy based on a patient's genomic profile.

\section{Conclusion}

The clinical evidence to date indicates that PAN is an important new addition to the treatment options for rrMM. The currently approved regimen using PAN in combination with bortezomib and dexamethasone has shown improvements in patient outcomes, including PFS and OS. The AEs noted to date are generally manageable with appropriate dosing strategies and supportive care. The ultimate role of PAN, beyond the approved regimen, remains unclear. Emerging data from the array of ongoing clinical trials are expected to provide additional insight regarding the place of PAN in the treatment of MM, including optimal dosing strategies and combination regimens.

\section{Acknowledgment}

The University of Michigan participated in the clinical trials which were funded by Novartis and involving panobinostat (CLBH589BUS69T, CLBH589E2214, CLBH589BUS58, CLBH5892203, CLBH589B2201, CLBH589B2211, and CLBH589B2203). The authors were not listed on the Form FDA1572 and were not members of the study team.

\section{Disclosure}

The authors report no conflicts of interest in this work.

\section{References}

1. Jagannath S, Dimopoulos MA, Lonial S. Combined proteasome and histone deacetylase inhibition: A promising synergy for patients with relapsed/ refractory multiple myeloma. Leuk Res. 2010;34(9):1111-1118.

2. Merin NM, Kelly KR. Clinical use of proteasome inhibitors in the treatment of multiple myeloma. Pharmaceuticals (Basel). 2014;8(1):1-20.

3. International Agency for Research on Cancer. Globoscan 2013: Estimated Cancer Incidence, Mortality, and Prevalence Worldwide in 2012. Available from: http://globocan.iarc.fr/Pages/fact_sheets_population. aspx. Accessed February 28, 2016.

4. Novartis Pharmaceutical Corporation. FDA Oncologic Drugs Advisory Committee Briefing Document: Panobinostat Capsules; 2014. Available from: http:/www.fda.gov/downloads/AdvisoryCommittees/ CommitteesMeetingMaterials/Drugs/OncologicDrugsAdvisoryCommittee/ UCM421624.pdf. Accessed November 1, 2015.

5. Laubach JP, Voorhees PM, Hassoun H, Jakubowiak A, Lonial S, Richardsom PG. Current strategies for treatment of relapsed/refractory multiple myeloma. Expert Rev Hematol. 2014;7(1):97-111.

6. Ashjian E, Redic K. Multiple myeloma: Updates for pharmacists in the treatment of relapsed and refractory disease. J Oncol Pharm Pract. 2016;22(2):289-302.

7. Libby E, Garcia D, Quintana D, et al. Disease-specific survival for patients with multiple myeloma: significant improvements over time in all age groups. Leuk Lymphoma. 2014;55(12):2850-2857.

8. National Comprehensive Cancer Network (NCCN). Clinical Practice Guidelines in Oncology: Multiple Myeloma. Version 2; 2016. Available from: http://www.nccn.org/professionals/physician_gls/pdf/myeloma. pdf. Accessed December 11, 2015.

9. Kumar DK, Therneau TM, Gertz MA, et al. Clinical course of patients with relapsed multiple myeloma. Mayo Clin Proc. 2004;79(7):867-874.

10. Moreau P. The future of therapy for relapsed/refractory multiple myeloma: Emerging agents and novel treatment strategies. Semin Hematol. 2012;49 Suppl 1:S33-S46.

11. Kaufman JL, Fabre C, Lonial S, Richardson PG. Histone deacetylase inhibitors in multiple myeloma: rationale and evidence for their use in combination therapy. Clin Lymphoma Myeloma Leuk. 2013;13(4):370-376.

12. Glozak MA, Seto E. Histone deacetylases and cancer. Oncogene. 2007; 26(37):5420-5432.

13. Pearl LH, Prodormou C. Structure and mechanism of the Hsp90 molecular chaperone machinery. Annu Rev Biochem. 2006;75:271-294.

14. Rosato RR, Alemnara JA, Grant S. The histone deacetylase inhibitor MS-275 promotes differentiation or apoptosis in human leukemia cells through a process regulated by generation of reactive oxygen species and induction of p21CIP1/WAF1 1. Cancer Res. 2003;63(13): 3637-3645. 
15. Xu W, Ngo L, Perez G, Dokmanovic M, Marks PA. Intrinsic apoptotic and thioredoxin pathways in human prostate cancer cell response to histone deacetylase inhibitor. Proc Natl Acad Sci US A. 2006;103(42): $15540-15545$.

16. Cea M, Cagnetta A, Gobbi M, et al. New insights into the treatment of multiple myeloma with histone deacetylase inhibitors. Curr Pharm Des. 2013;19(4):734-744.

17. Hideshima T, Bradner JE, Wong J, et al. Small-molecule inhibition of proteasome and aggresome function induces synergistic antitumor activity in multiple myeloma. Proc Natl Acad Sci U S A. 2005;102(24): $8567-8572$.

18. Felsenfield G, Groudine M. Controlling the double helix. Nature. 2003; 421(6921):448-453

19. Osada H, Tatematsu Y, Saito H, Yatabe Y, Mitsudomi T, Takahashi T. Reduced expression of class II histone deacetylase genes is associated with poor prognosis in lung cancer patients. Int J Cancer. 2004; 112(1):26-32.

20. Hideshima T, Richardson PG, Anderson KC. Mechanism of action of proteasome inhibitors and deacetylase inhibitors and the biological basis of synergy in multiple myeloma. Mole Cancer Ther. 2011;10(11): 2034-2042.

21. Adams, J. The proteasome: structure, function, and role in the cell. Cancer Treat Rev. 2003;29(Suppl 1):3-9.

22. Ellis L, Pan Y, Smyth GK, et al. Histone deacetylase inhibitor panobinostat induces clinical responses with associated alterations in gene expression profiles in cutaneous T-Cell lymphoma. Clin Cancer Res. 2008; 14(14):4500-4510.

23. Mitsiades N, Mitsaides CS, Richardson PG, et al. Molecular sequelae of histone deacetylase inhibition in human malignant B cells. Blood. 2003;101(10):4055-4062.

24. Catley L, Weisberg E, Kiziltepe T, et al. Aggresome induction by proteasome inhibitor bortezomib and alpha-tubulin hyperacetylation by tubulin acetylase (TDAC) inhibitor LBH589 are synergistic in myeloma cells. Blood. 2006;108(10):3441-3449.

25. Farydak ${ }^{\circledR}$ (panobinostat capsules) [package insert]. East Hanover, NJ: Novartis Pharmaceuticals Corporation; 2015.

26. DeAngelo DJ, Spencer A, Bhalla KN, et al. Phase Ia/II, two-arm, openlabel, dose-escalation study of oral panobinostat administered via two dosing schedules in patients with advanced hematologic malignancies. Leukemia. 2013;27(8):1628-1636.

27. WolfJL, SiegelD, Goldschmidt H, et al. A phase II trial of the pan-deacetylase inhibitor panobinostat as a single agent in advanced relapsed/refractory multiple myeloma. Leuk Lymphoma. 2012;53(9):1820-1823.

28. Ocio EM, Vilanova D, Atadja $\mathrm{P}$, et al. In vitro and in vivo rationale for the triple combination of panobinostat (LBH589) and dexamethasone with either bortezomib or lenalidomide in multiple myeloma. Haematologica. 2010;95(5):794-803.

29. San-Miguel JF, Richardson PG, Gunther A, et al. Phase Ib study of panobinostat and bortezomib in relapsed or relapsed and refractory multiple myeloma. J Clin Oncol. 2013;31(29):3696-3703.

30. Garnock-Jones KP. Panobinostat: first global approval. Drugs. 2015; 75:695-704.

31. San-Miguel JF, Hungria VT, Yoon SS, et al. Panobinostat plus bortezomib and dexamethasone versus placebo plus bortezomib and dexamethasone in patients with relapsed or relapsed and refractory multiple myeloma: a multicenter, randomized, double-blind phase 3 trial. Lancet Oncol. 2014;15(11):1195-1206.

32. Richardson PG, Schlossman RL, Alsina M, et al. PANORAMA 2: panobinostat in combination with bortezomib and dexamethasone in patients with relapsed and bortezomib-refractory myeloma. Blood. 2013; 122(14):2331-2337.

33. San-Miguel JF, Hungria VT, Yoon SS, et al. Efficacy and safety based on duration of treatment of panobinostat plus bortezomib and dexamethasone in patients with relapsed or relapsed and refractory multiple myeloma in the Phase 3 Panorama 1 study. Blood. 2014;124(21): 4742 .
34. San-Miguel JF, Hungria VT, Yoon SS, et al. Final analysis of overall survival from the phase 3 Panorama 1 trial of panobinostat plus bortezomib and dexamethasone versus placebo plus bortezomib and dexamethasone in patients with relapsed or relapsed and refractory multiple myeloma. Poster 3026, Session 653; presented at: American Society of Hematology 57th Annual Meeting and Exposition; December 2015; Orlando, FL.

35. San-Miguel JF, Hungria VT, Yoon SS, et al. Analysis of outcomes based on response in patients with relapsed or relapsed and refractory multiple myeloma treated with panobinostat or placebo in combination with bortezomib and dexamethasone in the Panorama 1 trial: updated analysis based on prior treatment. Poster 4230, Session 653; presented at: American Society of Hematology 57th Annual Meeting and Exposition; December 2015; Orlando, FL.

36. Reu FJ, Valent J, Malek E, et al. A phase I study of ixazomib in combination with panobinostat and dexamethasone in patients with relapsed or refractory multiple myeloma. Poster 4221, Session 653; presented at: American Society of Hematology 57th Annual Meeting and Exposition; December 2015; Orlando, FL.

37. Carfilzomib Plus Panobinostat in Relapsed/Refractory Multiple Myeloma (MM). Available from: https://clinicaltrials.gov/ct2/show/ NCT01301807?term=NCT01301807\&rank=1. Accessed December 11, 2015.

38. Kaufman JL, Zimmerman T, Rosenbaum CA, et al. Phase I study of the combination of carfilzomib and panobinostat for patients with relapsed and refractory myeloma: a Multiple Myeloma Research Consortium (MMRC) clinical trial. Blood. 2014;124(21):32.

39. Berdeja JG, Hart LL, Mace JR, et al. Phase I/II study of the combination of panobinostat and carfilzomib in patients with relapsed/refractory multiple myeloma. Haematologica. 2015;100(5):670-676.

40. Berdeja JG, Gregory TB, Faber EA, et al. A phase I/II study of the combination of panobinostat (PAN) and carfilzomib (CFZ) in patients (pts) with relapsed or relapsed/refractory multiple myeloma (MM): comparison of two expansion cohorts. Poster 1825, Session 653; presented at: American Society of Hematology 57th Annual Meeting and Exposition; December 2015; Orlando, FL.

41. Mateos M, Spencer A, Taylor K, et al. Phase Ib study of oral panobinostat (LBH589) plus lenalidomide (LEN) plus dexamethasone (DEX) in patients (pts) with relapsed (rel) or Rel and refractory (ref) multiple myeloma (MM). J Clin Oncol. 2010;28:15s. Abstr. 8030.

42. Chari A, Cho HJ, Len S, et al. A phase II study of panobinostat with lenalidomide and weekly dexamethasone in myeloma. Poster 4226, Session 653; presented at: American Society of Hematology 57th Annual Meeting and Exposition; December 2015; Orlando, FL.

43. A Phase I Study of Panobinostat/Lenalidomide/Bortezomib/Dex for Relapsed and Relapsed/Refractory Multiple Myeloma (PanRVD). Available from: https://clinicaltrials.gov/ct2/show/NCT01965353? term=NCT01965353\&rank=1. Accessed December 11, 2015.

44. Combination Therapy of Lenalidomide/Bortezomib/Dexamethasone and Panobinostat in Transplant Eligible New Diagnosed Multiple Myeloma (MM) Patients. Available from: https://clinicaltrials.gov/ ct $2 /$ show $/$ NCT01440582?term $=$ NCT01440582\&rank $=1$. Accessed December 11, 2015.

45. Popat R, Brown S, Flanagan LM, Cavenagh JD. Velcade, thalidomide, dexamethasone and panobinostat (VTD-P) for patients with relapsed and relapsed/refractory myeloma: preliminary results of the Muk-Six phase I/IIa trial. Blood. 2014;124(21):4766.

46. Popat R, Brown SR, Flanagan LM, et al. Bortezomib (Velcade), thalidomide, dexamethasone and panobinostat (VTD-P) is a safe, well tolerated and efficacious regimen for patients with relapsed multiple myeloma: preliminary results of the Muk-Six trial. Poster 1826, Session 653; presented at: American Society of Hematology 57th Annual Meeting and Exposition; December 2015; Orlando, FL.

47. Panobinostat and Everolimus in Treating Patients with Recurrent Multiple Myeloma, non-Hodgkin Lymphoma, or Hodgkin Lymphoma. Available from: https://clinicaltrials.gov/ct2/show/NCT00918333? term=nct00918333\&rank=1. Accessed December 11, 2015. 
48. Bobrowicz M, Dwojak M, Malenda A, et al. HDAC inhibitors as potential new agents improving the efficacy of monoclonal antibodies. Blood. 2014;124(21):3641.

49. Panobinostat Combined With High-Dose Gemcitabine/Busulfan/ Melphalan With Autologous Stem Cell Transplant for Patients With Refractory/Relapsed Myeloma. Available from: https://clinicaltrials. gov/ct2/show/NCT02506959?term=nct02506959\&rank=1. Accessed December 11, 2015.

50. Richardson PG, Hungria VTM, Yoon SS. Characterization of the incidence and management of gastrointestinal toxicity in the phase 3 Panorama 1 study of panobinostat plus bortezomib and dexamethasone versus placebo plus bortezomib and dexamethasone in patients with relapsed or relapsed and refractory multiple myeloma. Blood. 2014; 124(21):2120.

51. Hansen VL, Coleman M, Elkins S, et al. An expanded treatment protocol of panobinostat plus bortezomib and dexamethasone in patients with previously treated myeloma. Poster 3027, Session 653; presented at: American Society of Hematology 57th Annual Meeting and Exposition; December 2015; Orlando, FL.
52. Richardson PG, Lee JH, Majer I, Krishna A, Woodman RC. Efficacy of treatments in relapsed or relapsed and refractory multiple myeloma: an indirect treatment comparison. Blood. 2014;124(21):2638.

53. Ruggeri K, Maguire A, Schmitz S, et al. Estimating the relative effectiveness of treatments in relapsed/refractory multiple myeloma through a systematic review and network meta-analysis. Poster 2103, Session 902; presented at: American Society of Hematology 57th Annual Meeting and Exposition; December 2015; Orlando, FL.

54. Radomska H, Canella A, Jessica C, et al. Small RNA deep sequencing highlights the important contribution of MiRNAs in regulating IRF4/c-Myc axis in myeloma development. Poster 1791, Session 651; presented at: American Society of Hematology 57th Annual Meeting and Exposition; December 2015; Orlando, FL.

55. U.S. National Institutes of Health. Clinical Trials Registry. Available from: https://clinicaltrials.gov/. Accessed December 14, 2015.
OncoTargets and Therapy

\section{Publish your work in this journal}

OncoTargets and Therapy is an international, peer-reviewed, open access journal focusing on the pathological basis of all cancers, potential targets for therapy and treatment protocols employed to improve the management of cancer patients. The journal also focuses on the impact of management programs and new therapeutic agents and protocols on

\section{Dovepress}

patient perspectives such as quality of life, adherence and satisfaction. The manuscript management system is completely online and includes a very quick and fair peer-review system, which is all easy to use. Visit http://www.dovepress.com/testimonials.php to read real quotes from published authors. 